\title{
Gravitational waves and core-collapse supernovae
}

\section{S.G. Moiseenko*}

Space Research Institute, Profsoyuznaya str. 84/32, Moscow 117997, Russia

E-mail: moiseenko@iki.rssi.ru

\section{G.S.Bisnovatyi-Kogan}

Space Research Institute, Profsoyuznaya str. 84/32, Moscow 117997, Russia

Research Nuclear University "MEPHI", Kashirskoye Shosse, 31, Moscow 115409, Russia

E-mail: gkoganeiki.rssi.ru

\begin{abstract}
Numerical simulation Results of 2D magnetorotational core-collapse supernova explosions are presented. These results are used to estimate the dimensionless amplitude of the gravitational wave with a frequency $v \sim 1300 \mathrm{~Hz}$, radiated during the collapse of the rotating core of a presupernova with a mass of $1.2 M_{\odot}$. This estimate agrees well with many other calculations that have been done in 2D and 3D settings and which rely on more exact and sophisticated calculations of the gravitational wave amplitude.
\end{abstract}

Multifrequency Behaviour of High Energy Cosmic Sources - XIII - MULTIF2019

3-8 June 2019

Palermo, Italy

${ }^{*}$ Speaker. 


\section{Introduction}

One of the complicated and not solved to the end problems of modern astrophysics is clarification of the physical mechanism of core collapse supernova explosion. One dimensional ( spherically symmetrical) simulations of the problem did not succeed. Main forces today are directed to $2 \mathrm{D}$ and $3 \mathrm{D}$ numerical models.

Physical mechanism of core collapse supernova explosion based on neutrino radiation (neutrino driven supernovae) was simulated numerically many times with sophisticated physics (detailed calculations of neutrino transport including neutrino oscillations, modern equations of state). But up to know these simulations either did not lead to the explosion, or the explosion supernova energy was not enough to fit observational data.

The core-collapse supernova neutrino driven explosions are numerically simulated by many scientific groups in the world (see for example, [1] and reference therein).

In 1970 G.S.Bisnovatyi-Kogan [2] suggested physical mechanism of core collapse supernova which takes into account rotation and magnetic field. The idea of the magnetorotational(MR) mechanism consists of angular momentum transfer outwards using magnetic field, which is twisting due to the differential rotation. The toroidal component of the magnetic field is amplifying with time what leads to increasing of the magnetic pressure and generating the supernova shock. The MR supernovae explosions are always asymmetrical hence the gravitational waves(GW) will be emitted in the process of the core collapse. In the paper we estimate the GW signal from our numerical simulations of MR supernovae. This estimate agrees well with many other calculations that have been done in 2D and 3D settings and which rely on more exact and sophisticated calculations of the gravitational wave amplitude.

\section{Magnetorotational supernova explosion}

We use set of MHD equations with infinite conductivity and self-gravitation:

$$
\begin{gathered}
\frac{\mathrm{d} \mathbf{x}}{\mathrm{d} t}=\mathbf{u}, \quad \frac{\mathrm{d} \rho}{\mathrm{d} t}+\rho \operatorname{div} \mathbf{u}=0, \\
\rho \frac{\mathrm{d} \mathbf{u}}{\mathrm{d} t}=-\operatorname{grad}\left(p+\frac{\mathbf{H} \cdot \mathbf{H}}{8 \pi}\right)+\frac{\operatorname{div}(\mathbf{H} \otimes \mathbf{H})}{4 \pi}-\rho \operatorname{grad} \Phi \\
\rho \frac{\mathrm{d}}{\mathrm{d} t}\left(\frac{\mathbf{H}}{\rho}\right)=\mathbf{H} \cdot \nabla \mathbf{u}, \quad \Delta \Phi=4 \pi G \rho, \\
\rho \frac{\mathrm{d} \varepsilon}{\mathrm{d} t}+p \operatorname{div} \mathbf{u}=F_{V}(\rho, T), \quad P=P(\rho, T), \quad \varepsilon=\varepsilon(\rho, T),
\end{gathered}
$$

where $\frac{\mathrm{d}}{\mathrm{d} t}=\frac{\partial}{\partial t}+\mathbf{u} \cdot \nabla$ - full time derivative, $\mathbf{x}=(r, \varphi, z), \mathbf{u}$ - velocity vector, $\rho$ - density, $p$ - pressure, $\mathbf{H}=\left(H_{r}, H_{\varphi}, H_{z}\right)$-magnetic field, $\Phi$ - gravitational potential, $\varepsilon$ - internal energy, $G$ - gravitational constant, $\mathbf{H} \otimes \mathbf{H}$ - rank 2 tensor. $F_{V}(\rho, T)$ - neutrino losses.

We suppose axial symmetry $\left(\frac{\partial}{\partial \varphi}=0\right)$ and equatorial symmetry to the equatorial plane $(z=0)$. 
Simulation of the problem was done in bounded area. Outside the area the density of the matter was expected equal zero $(\rho=0)$, while the poloidal components of the magnetic filed $H_{r}, H_{z}$ could be nonzero.

Additional condition - divergence-free of the magnetic field $\operatorname{div} \mathbf{H}=0$.

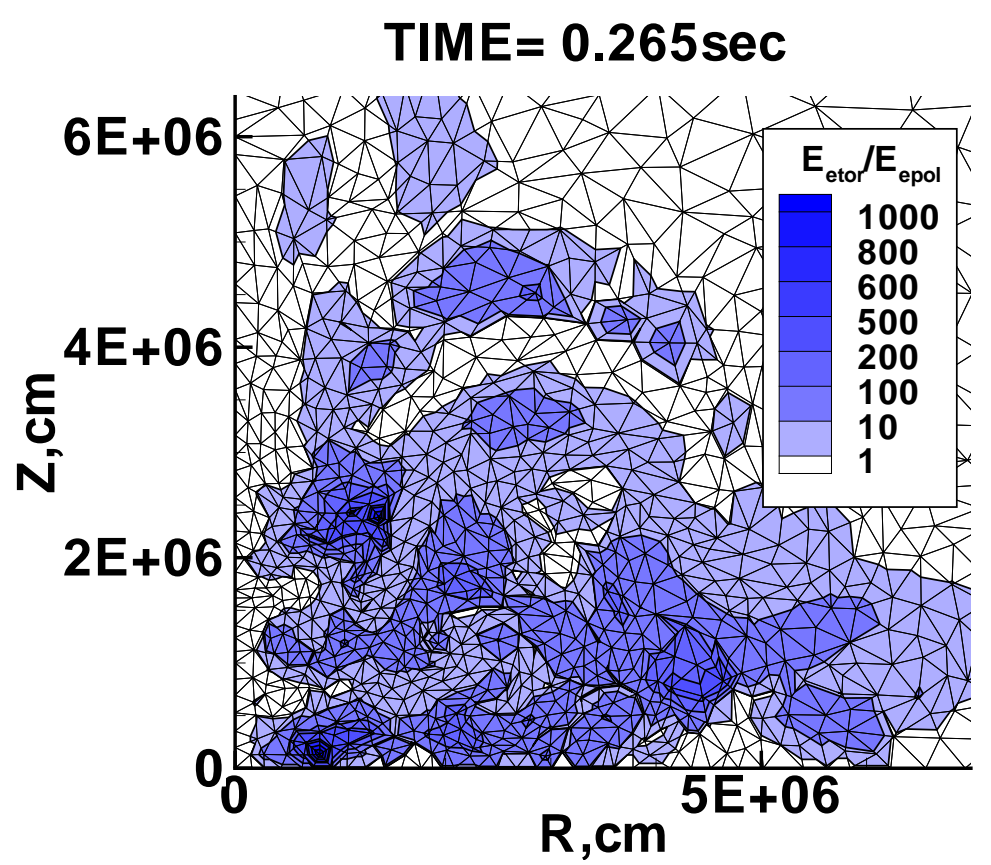

Figure 1: The Lagrangian triangular grid and the ratio of the toroidal magnetic energy $E_{t o r}$ to the poloidal magnetic energy $E_{p o l}, \frac{E_{t o r}}{E_{p o l}}$ at $t=265 \mathrm{~ms}$ for the case $H_{0}=10^{9} \mathrm{G}, E_{\text {rot } 0} / E_{\text {grav } 0}=1 \%$.

MR explosion with the initial quadrupole-like magnetic field was described in detail in the paper [3]. After the core collapse the pre SN rotates differentially. The toroidal component of the magnetic field appears and grows linearly with time at the initial stage of the MR explosion. When the toroidal magnetic field reaches some certain value its linear growth changes to the exponential growth of the toroidal and poloidal components due to the onset of the MDRI. A toy model for the qualitative explanation of the MDRI in MR supernova was suggested in [3]. MR supernova explosion with initial quadrupole-like magnetic field results in explosion which develops mainly near equatorial plane.

Simulations of the MR supernova with the initial magnetic field of dipole-like symmetry leads to the qualitatively different result in the shape of explosion [4]. In this case the MR explosion develops mainly along the axis of rotation and forms mildly collimated proto jet.

The protojet found in our simulations could be collimated when it develops in the extended envelope of the massive star - the progenitor of the core collapse supernova.

MDRI leads to the formation of chaotic magnetic field structure. In the case of finite conductivity the reconnection of the magnetic field could be important. We have estimated characteristic time of the reconnection of the magnetic field using results of our simulations [4]. We found that characteristic time for the reconnection of the magnetic field for supernova parameters used in our simulations is $\approx 5 \mathrm{~s}$. The MR supernova explosion time in our simulations is $\approx 0.5-1 \mathrm{~s}$ what is 


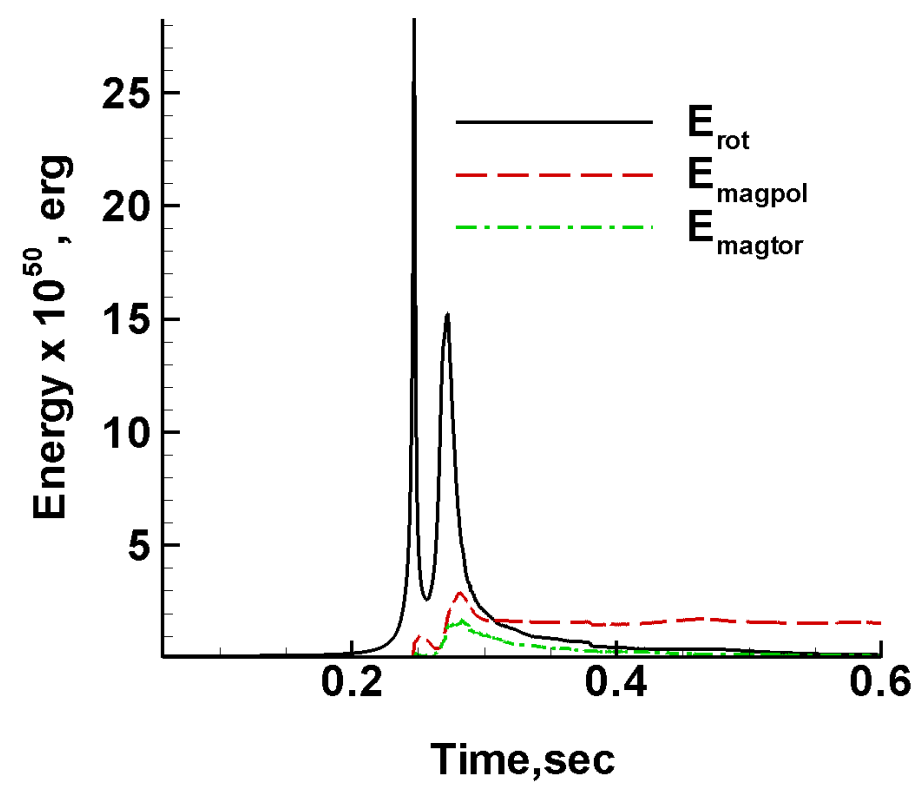

Figure 2: Time evolution of rotational energy $E_{\text {rot }}$ (solid line), magnetic poloidal energy $E_{\text {magpol }}$ (dashed line) and magnetic toroidal energy $E_{\text {magtor }}$ (dash-dotted line) for the case $H_{0}=10^{9} \mathrm{G}, E_{\text {rot } 0} / E_{\text {grav } 0}=1 \%$.

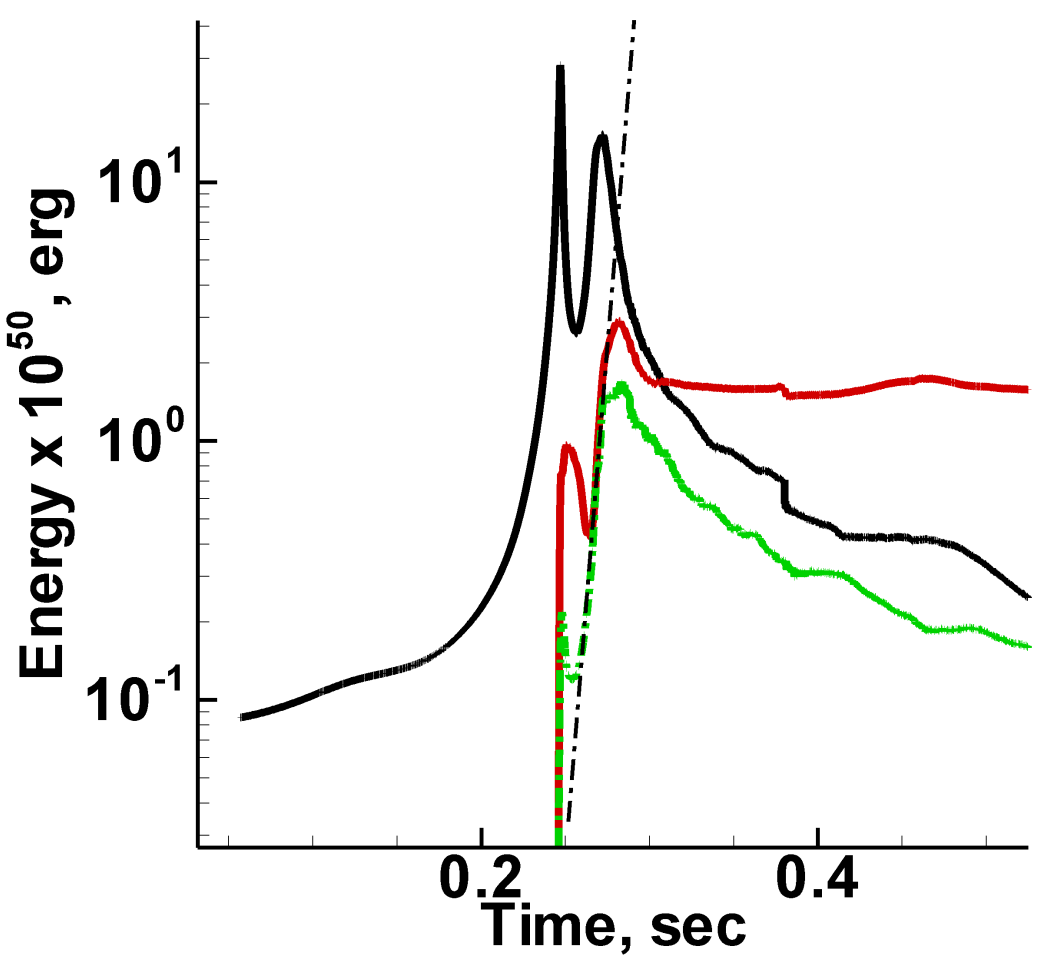

Figure 3: Zoomed time evolution of rotational energy $E_{\text {rot }}$ (solid line), magnetic poloidal energy $E_{\text {magpol }}$ (dashed line) and magnetic toroidal energy $E_{\text {magtor }}$ (dash-dotted line) for the case $H_{0}=10^{9} \mathrm{G}, E_{\text {rot } 0} / E_{\text {grav } 0}=$ $1 \%$. Straight dash-dotted line shows exponential growth of the toroidal and poloidal magnetic energies. 
significantly less then characteristic time of the magnetic field reconnection development, and do not influence the MR supernova significantly.

At the plots we briefly represent results of our simulations of magnetorotational supernova explosion from [3, 4]

At Fig. 1 The Lagrangian triangular grid and the ratio of the toroidal magnetic energy to the poloidal one $\left(E_{t o r} / E_{p o l}\right.$ is represented for the case $\left(H_{0}=10^{9}, \mathrm{G}\right)$ and $E_{\text {rot } 0} / E_{\text {grav } 0}=1 \%$. The toroidal magnetic energy dominates over the poloidal one in the significant part of the region where new neutron star is forming. The MDRI is well-resolved on our triangular grid.

The Fig. 2 represents a time evolution of rotational, magnetic poloidal and toroidal energies for MR explosion when $H_{0}=10^{9} \mathrm{G}$.

The Fig. 3 is the same data plot as the Fig. 2 but zoomed and the vertical axis is in logarithmic scale. The straight dash-dotted line at the Fig.3 shows the exponential growth of the toroidal and poloidal magnetic energies with the time due to the MDRI.

The Fig.4 shows the time evolution of the magnetic field components as an example of MDRI development.

We have done simulations for different initial masses of the iron core and for different initial angular velocities of presupernova. The supernova explosion energy grows significantly with increase of the core mass. The dependence of the explosion energy on the core mass for the different initial values of the rotational energy (angular velocity) is presented at Fig.5 for the initial quadrupole-like magnetic field [5].

\section{Gravitational signal from core collapsed supernova}

Results of multidimensional simulations of core-collapse supernovae were used to calculate the emitted gravitational energy and the form of the gravitational waves $(\mathrm{GW})$ signal. The gravitational signal from nonspherical gravitational collapse was calculated in [6, 7]. Ones of the first papers where the gravitational signal was calculated using the results of the numerical simulations of core collapse supernova are $[8,9]$. To calculate the GW power $\dot{E}$ in numerical simulations the quadrupole moment

$$
D_{\alpha \beta}=\int \rho(\mathbf{r}, t)\left(3 r_{\alpha} r_{\beta}-\delta_{\alpha \beta} r^{2}\right)
$$

an its time derivatives are calculated. Then the $\dot{E}$ is calculated by formula [10]

$$
\dot{E}=\frac{G}{45 c^{5}} \dddot{D}_{\alpha \beta} \dddot{D}^{\alpha \beta}
$$

Papers $[11,12]$ represent the detailed calculations of the gravitational signal for rotation core collapse supernovae simulations. It was shown that the GW signal frequency is about $700 \mathrm{~Hz}$ for the specified equation of state.

In the paper [13] the gravitational signal from nonspherical collapse of rigidly rotating dust cloud was studied. The gravitational signal from oblate spheroid of a mass $M$, major and minor semi-axes $A$ and $C$ correspondingly was considered. The gravitational power can be expressed by the formula:

$$
L_{G W}=\frac{2}{375} \frac{G M^{2}}{c^{2}}\left(A^{2}-\dddot{C}^{2}\right)^{2} .
$$



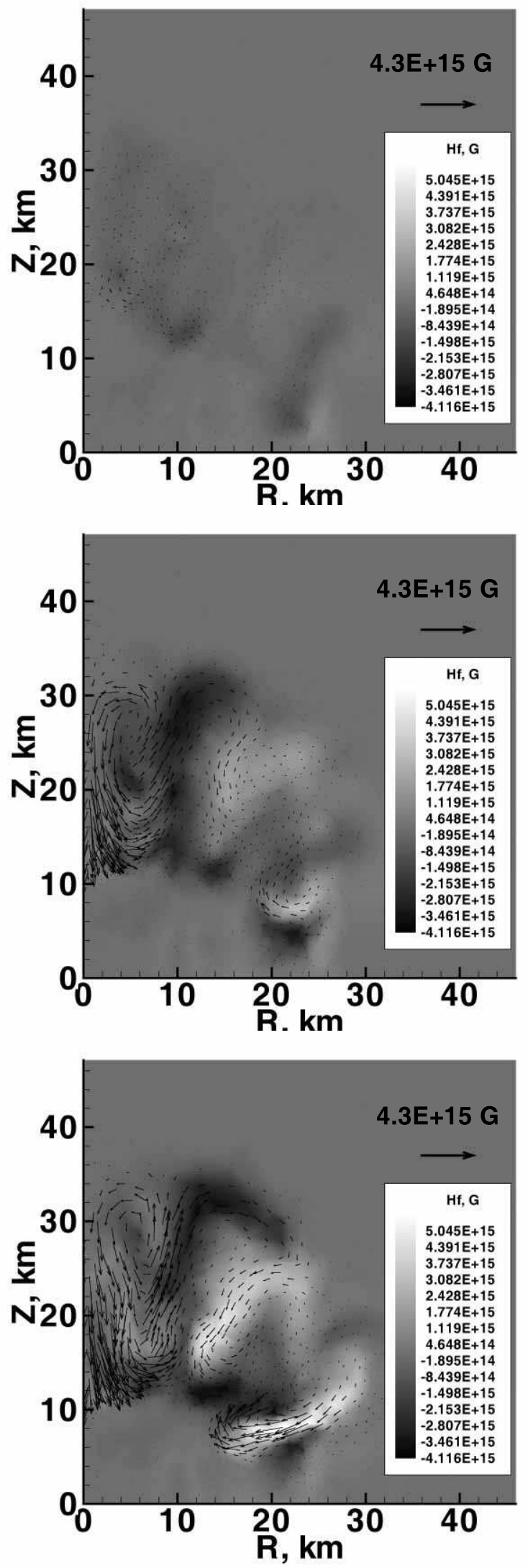

Figure 4: An example of the MDRI development in MR supernova explosion . Gray scale is the toroidal field $H_{\varphi}$ levels. Arrows show a direction and strength of the poloidal magnetic field $H_{r}, H_{z}$ [4]. 


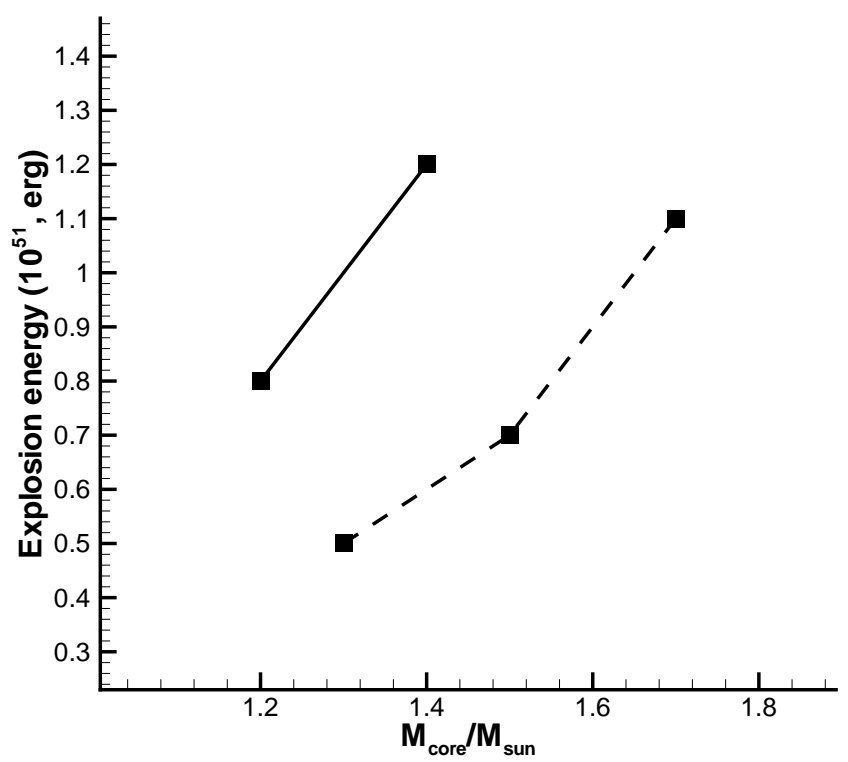

Figure 5: Dependence of the supernova explosion energy on the core mass for initial angular velocity $\omega_{0}$ $\approx 3.53 s^{-1}$ (solid line) and $\omega_{0} \approx 2.52 s^{-1}$ (dashed line) (before collapse).

In the paper [14] it was found that main part the GW energy is emitted during bounce process. To avoid singularity (infinite density) in the collapse of dust matter at bounce moment the ellipsoid with the finite entropy was considered, its density and thickness remain finite at the bounce time. In [14] the following formula was suggested:

$$
E_{G W} \approx k M c^{2}\left(r_{g} / A_{\min }\right)^{7 / 2} \frac{A_{\min }}{C_{\min }}
$$

where $r_{g}=2 G M / c^{2}$ - Schwarzschild radius, $A_{\min }$ - minimal value of major semi-axis, $A_{\min }$ - minimal value of minor semi-axis.

According to [14] $k \sim 0.01$.

We roughly estimate the gravitational wave power from our simulations done in [15] for the core mass is $1.2 M_{\odot}[16]$.

From formula (3.4) we get the energy of GW for the following calculated bounce parameters:

$$
\begin{aligned}
& A_{\min }=5.5 \cdot 10^{5} \mathrm{~cm}, \quad \frac{C_{\min }}{A_{\min }}=0.5 \quad \text { for } \quad M=0.24 M_{\odot}, \quad E_{G W}=1.3 \cdot 10^{49} \mathrm{erg}, \\
& A_{\text {min }}=5.3 \cdot 10^{6} \mathrm{~cm}, \quad \frac{C_{\min }}{A_{\min }}=0.79 \quad \text { for } \quad M=0.3 M_{\odot}, \quad E_{G W}=1.2 \cdot 10^{46} \mathrm{erg}, \\
& A_{\min }=9.5 \cdot 10^{6} \mathrm{~cm}, \quad \frac{C_{\min }}{A_{\min }}=0.83 \quad \text { for } \quad M=0.5 M_{\odot}, \quad E_{G W}=5.4 \cdot 10^{45} \mathrm{erg}, \\
& A_{\min }=3 \cdot 10^{7} \mathrm{~cm}, \quad \frac{C_{\min }}{A_{\min }}=0.89 \quad \text { for } \quad M=0.8 M_{\odot}, \quad E_{G W}=7.4 \cdot 10^{44} \mathrm{erg}, \\
& A_{\min }=4.2 \cdot 10^{7} \mathrm{~cm}, \quad \frac{C_{\min }}{A_{\min }}=0.94 \quad \text { for } \quad M=M_{\odot}, \quad E_{G W}=5.9 \cdot 10^{44} \mathrm{erg} .
\end{aligned}
$$


Here $M$ is star mass inside constant density surface. The energy of the emitted GW is approximately equal to the maximal value of the $\left(E_{G W}=1.3 \cdot 10^{49} \mathrm{erg}\right.$ for the mass $\left.M=0.24 M_{\odot}\right)$ in (3.6). This value is defined by the mass inside the isodence with maximal compression.

The relative amplitude of gravitational wave $h_{\alpha \beta}$ at the distance $r$ for collapsing object is defined by the formula $[10,17]$

$$
h_{\phi \phi}=-h_{\theta \theta}=\frac{G M}{5 r c^{4}} \sin ^{2} \theta_{0}\left(\ddot{a}^{2}-\ddot{c^{2}}\right), \quad h_{\theta \phi}=0 .
$$

Here the angle $\theta_{0}$ is calculated from $z$ axis of spheroid. To estimate the maximum of the amplitude in relation to the observational angle we can write approximately (3.6) for the dimensionless amplitude of the GW at $r$ distance form the source we have

$$
h_{\phi \phi}=-h_{\theta \theta}=\frac{G M}{5 r c^{4}} \frac{A_{\min }^{2}}{(\Delta t)^{2}} .
$$

Using (3.6) for $M=0.24 M_{\odot}$, we get

$$
h=h_{\phi \phi}=-h_{\theta \theta}=\frac{6.7 \cdot 10^{-8} \cdot 0.48 \cdot 10^{33}}{5 r c^{4}} \frac{\left(5.5 \cdot 10^{5}\right)^{2}}{\left(0.75 \cdot 10^{-3}\right)^{2}} \approx 1.4 \cdot 10^{-22} \frac{r}{10 \mathrm{kpc}} .
$$

\section{Conclusions}

The relative amplitude $h$ of gravitational waves produced by core-collapse supernova at a distance of $10 \mathrm{kpc}$ is $\sim 10^{-22}-10^{-20}$ The frequency of the signal is about $1000 \mathrm{~Hz}$. The GW signal of such a high amplitude can be registered by modern gravitational detectors.

This work was partially supported by RFBR grant No.18-02-00619.

\section{References}

[1] Janka, H.-T. 2017, Handbook of Supernovae, 1095

[2] Bisnovatyi-Kogan G.S., 1970, Astron. Zh. 47, 813 (Soviet Astronomy, 1971, 14, 652)

[3] Ardeljan N.V., Bisnovatyi-Kogan G.S., Moiseenko S.G., 2005, MNRAS, 359, 333

[4] Moiseenko S.G., Bisnovatyi-Kogan G.S., Ardeljan N.V., 2006, MNRAS, 370, 501

[5] Bisnovatyi-Kogan, G. S., Moiseenko, S. G., \& Ardelyan, N. V. 2008, Astronomy Reports, 52, 997

[6] Schutz B.F. Class. Quantum Grav. 6, 1761 (1989)

[7] Smith S.C., Houser J.L., Centrella J.M. Astrophys. J. 458, 236 (1996)

[8] Sazhin M.V., Ustyugov S.D., Chechetkin V.M. JETP Lett 64, 871 (1996)

[9] Sazhin M.V., Ustyugov S.D., Chechetkin V.M. JETP 86, 629 (1998)

[10] Landau L.D., Lifshitz E.M. The classical theory of fields (Oxford:, Butterworth-Heinemann, 2000)

[11] Dimmelmeier H., Ott C.D., Janka H.-T., Marek A., Mueller E. Phys. Rev. Lett. 98, 251101 (2007)

[12] Dimmelmeier H., Ott C.D., Marek A., Janka H.-T. Physical Review D 78, 064056 (2008)

[13] Thuan T.X., Ostriker J.P. ApJL, 191 L105, (1974) 
[14] Novikov I.D. Sov. Astron. 19 398, (1976)

[15] Ardeljan N.V., Bisnovatyi-Kogan G.S., Moiseenko S.G. MNRAS 359, 333 (2005)

[16] Bisnovatyi-Kogan, G. S., \& Moiseenko, S. G. Physics Uspekhi, 60, 84, (2017)

[17] Saenz R.A., Shapiro S.L. Astrophysical Journal 221, 286 (1978)

\section{DISCUSSION}

JAMES H. BEALL: Did you take magnetic reconnection into account in your simulations?

SERGEY MOISEENKO: We did not take into account magnetic reconnection in our simulations while we have estimated the time scale for the magnetic reconnection in our case. Rough estimations show that the reconnection time scale is about 5 seconds. The magnetorotational explosion develops in less than 0.5 seconds. 